\title{
Designer Driving: Drivers' Conceptual Models and level of trust in Adaptive -Cruise -Control
}

\author{
Tarannum. Ayesha Kazi ${ }^{a^{*},}{ }^{b}$ Neville A. \\ Stanton, ${ }^{\mathrm{C}}$ Guy H. Walker \& ${ }^{\mathrm{d}}$ Mark S. Young \\ ${ }^{a^{*}}$ TRL Crowthorne House, Nine Mile Ride, Wokingham, Berkshire, \\ RG40 3GA, UK \\ b, c, d School of Engineering \& Design, Brunel University, Uxbridge, \\ Middlesex, UB8 3PH, UK
}

\begin{abstract}
A longitudinal driving simulator study was completed in order to examine drivers' level of trust and the type of conceptual model they held of an Adaptive-Cruise-Control system (ACC). Fifteen drivers participated in a ten day study where they were allocated to one of three ACC conditions. This was either a reliable ACC condition (100\%), semi- reliable ACC condition (50\%) or an unreliable ACC condition ( $0 \%)$. Results demonstrated that self-reported trust levels in the ACC system rose over time for the reliable group. Overall the reliability of automation did influence the ratings of trust and there was also evidence that drivers' self-reported trust was inappropriate in relation to the corresponding level of reliability. This may have implications for driver trust models, especially if these models have been forged after one-off short-term experimentation. In addition the drivers' conceptual models were consolidated over a short period of time, however they did not match that of designers' model of Adaptive -Cruise - Control, thus better design solutions may be warranted.
\end{abstract}

Keywords: Adaptive-Cruise- Control; Trust; Drivers' Conceptual Models; Longitudinal study.

Reference to this paper should be made as follows: Kazi, T.A, Stanton, N.A., Walker, G.H., \& Young, M.S. (200x)`Designer Driving: Drivers’ Conceptual Models and level of trust in Adaptive -Cruise -Control, Vol. X, No. Y,pp.000000 .

Biographical Notes: Dr Tara Kazi interests lie in Human Factors; Smart Computing Technologies (Pervasive, Affective and Wearable Computing) and in the broad area of Traffic Psychology. Before joining TRL, Tara worked at Brunel University, for the ERG (Ergonomics Research Group).

\section{Introduction}

\subsection{Adaptive-Cruise Control}

Adaptive Cruise Control (ACC) is, arguably, at the vanguard of future driver automation systems. When activated, ACC maintains a desired road speed and a constant time gap from a moving vehicle ahead, without the driver having to use the accelerator or brake. ACC is ostensibly a "comfort and convenience” system (Richardson et al, 1997), but inevitably, drivers' “[...] change their characteristics; they adapt to the functional characteristics of the working system, and they modify system characteristics to serve 
their particular needs and preferences” (Rasmussen et al., 1994). Driver workload and driving performance, two safety critical variables, have been reported to be influenced when driving with ACC. For instance, drivers report that driving with ACC is less effortful compared to driving manually (Hoedemaeker and Brookhius, 1998) and as a consequence driving with ACC may reduce driver vigilance and increase driver distraction (Stanton, Young, and McCaulder, 1997). Furthermore, drivers tend to brake harder, more often and later when driving with ACC (Hoedemaeker and kopf, 2001; Sayer, Franscher, Bareket \& Johnson, 1995) and there is an increase in lane position variability (Hoedemaeker and Brookhius, 1998).

One of the other critical variables involved in this adaptation is the concept of trust and consequent driver reliance on ACC. Lee and See (2004) suggest that the appropriateness of trust should be considered in relation to the context of the environment and goals of the user. For instance it can be argued that engaging the ACC during bad weather conditions is misuse of the system and is indicative of over reliance. Thus trust is often described as an attitude and reliance is perceived as a behaviour (Lee \& See, 2004). The aim of this paper is to explore ACC from a human perspective and to examine the relationship between system characteristics and the mechanisms of trust.

The 'problems' and 'ironies' of ACC stem from its credentials as a 'comfort and convenience' system. The system is powerful enough to relieve the driver of routine tasks, but not sufficiently powerful to assist the driver in situations of high demand; it is, as Norman (1990) states, at an intermediate level of intelligence. The irony of this form of automation (Bainbridge, 1974) is that in some situations it can engender a state of mental under load (Young and Stanton, 2001), to the extent that the driver's ability to intervene and to switch modes from supervisory control to full manual control, is significantly hampered (e.g. Stanton, Young, and McCaulder, 1997).

Conversley, Rudin-Brown and Parker (2004) show how drivers adapt to the functional characteristics of the system in a similar way to how they have been shown to adapt to Anti Lock Brakes (Wilde, 1982). They tend to brake harder, use minimum headway settings and drive at higher speeds. Clearly there is some 'utility' to be gained by this behaviour (at least from a Risk Homeostasis perspective, where drivers supposedly drive to match an internal target level of risk, Wilde, 1982,) but it is undeniably a case of automation 'misuse' (Parasuraman and Riley, 1997) as well as a case of behavioural adaptation. The research question, evidently, is one of attempting to engender the correct behavioural adaptation to the system, to stimulate a usage of it that is appropriate to its capabilities. The key to these issues lies in a range of intervening variables, ranging from mental workload and situational awareness through to mental models and personality. The concept of trust, it is argued, is particularly cogent and is selected as a candidate for further examination.

\subsection{The dynamics of trust in automation}

Trust can often determine the use of automated systems (Muir and Moray, 1996). However Lee and See (2004) suggest that trust can guide but does not determine reliance upon automation. Factors that influence trust within automation systems are: the reliability of the system, self -confidence, familiar or unfamiliar environments, and the individual's Locus of Control (Muir, 1994; Lee and Moray, 1992; Moray, Inagaki and Itoh, 2000; Lewandowsky, Mundy, and Tan, 2000; Kantowitz, Hanowski and Kantowitz, 
1997; Stanton and Young, 2005). Therefore increased unreliability of an automation system can lead to a decrease in the employment of the system (Muir, 1994; Lee \& Moray, 1992; Kantowitz et al, 1997). Lee and See (2004) argue that it is critical that a human operator's level of trust is calibrated to an appropriate level to match the genuine capabilities of automation to avoid misuse and disuse of automation.

Trust has been shown to influence driving performance with ACC as demonstrated by Rudin-Brown and Parker (2004). The results indicated that drivers' ratings of trust in the ACC increased after using the system and were unaffected by the malfunction of the ACC when set at the long gap settings. Rudin-Brown and Parker (2004) suggest that the degree of behavioural adaptation will be directly related to the trust a driver has for the ACC.

Experience and practice would improve trust and performance over time in ACC since the work of Muir (1994) and Muir and Moray 1996) recommends that operators should be given experience with automation in order to re-calibrate their trust when they discover the systems' properties. Certainly the advantage of Rudin-Brown and Parker's (2004) study was that the behavioural adaptation of drivers to ACC was assessed via an on-road demonstration, but since it was not a longitudinal approach, it is feasible that drivers may have calibrated their trust in ACC to a more appropriate level.

In addition, the results of Rudin-Brown and Parker's (2004) study highlight the type of conceptual model drivers have of the ACC system. It is plausible that drivers may not have fully comprehended the correct functioning or the limitations of the ACC system; this may explain the rise of trust in a malfunctioning ACC system.

\subsection{Drivers' Conceptual Model of ACC}

Preece, Rogers and Sharp (2002) describe a conceptual model (CM) as mental representations or metaphors that allows the user to interpret the device's behaviour. There are at least three interacting components: the designer, the user, and the system. Underlying these components are three interlinking CM's; the design model: which is the model the designer has of how the system should function; the System Image: how the system actually functions; the user's model: how the user understands the functioning of the system. Ideally the designers should design usable systems with clear system images that convey to the user how to employ the system. If the system image fails to clarify the design model to the users, however, then the users can potentially develop an incorrect understanding of the system, which in turn will make them use the system ineffectively and errors will arise (Preece et al, 2002).

Users of a system are also at risk of constructing appropriate conceptual models due to the practice known as confirmation bias. This is where people overlook contradictory information, in a bid to save cognitive resources (Besnard, Greathead, and Baxter, 2004). Rudin-Brown and Parker (2004) employed drivers that had experience with conventional cruise control (CC) thus this prior knowledge of the CC system combined with confirmation bias may have confounded the correct construction of a conceptual model of the ACC.

The drivers' conceptual model of the ACC system and driver's trust in the system could have implications for driver safety, driving performance and system design. For instance if drivers' have a tendency to over rely on the system because they have misunderstood the functional parameters of the ACC design, they may have a tendency to 
engage the system in situations where the ACC is not designed to cope, such as during bad weather conditions (as the radar sensors may fail) and in high traffic congestion environments (since the ACC switches to manual mode when driving speed falls below $16 \mathrm{mph}$. However much of the experimentation on trust in ACC and driving performance has been shaped by short-term, one-off experiments (such Rudin-Brown and Parker, 2004 and Hoedemaeker and Brookhius, 1998) not allowing for the possible recalibration of trust over time, or developing experience and familiarity with the system. Further, short-term experimentation may not necessarily contribute very much to an enlightened view of driver's conceptual models of the system, compared to longitudinal studies.

Hence a longitudinal study was carried out to examine the drivers' performance, level of trust and conceptual model of the system. Two additional novel approaches were undertaken to further assess drivers' trust in ACC and conceptual models, in the form of AGNA (a social network analysis tool) and a diary method. The experimental hypothesis of the study expected experience of driving with ACC and reliability of ACC would influence drivers' ratings of trust and conceptual models of ACC.

\section{METHOD}

\subsection{Design}

Trust and conceptual models were measured in the current study. Trust was measured using a ten item rating scale, whereas conceptual models were measured using a pictorial model building exercise. The study was based around a mixed design. The between subjects variable was automation reliability, with three levels (100\% reliable, reliable only $50 \%$ of the time, and reliable $0 \%$ of the time). A two-tailed prediction denoted that there would be a difference in the levels of trust between the three groups of automation conditions. The within subjects variable was time, measured at 10 consecutive time intervals ( 1 to 10 days). A two-tailed prediction denoted that there would be a difference in the levels of trust for each group over time and a difference in the drivers' conceptual models between the three groups and over time.

\subsection{Participants}

Fifteen Brunel University students (ten males and five females), aged between 18-26 (mean age 23), who had no experience with ACC or CC participated in the study. All participants held a valid driver's license (a mean of two years), drove regularly and were remunerated for their participation

\subsection{Apparatus}

2.3.1Driving Simulator The Brunel Driving simulator (medium fidelity) comprised a Jaguar XK8 type (see figure 1 below). The driver is presented with a three-lane motorway on a projection screen viewed through the windscreen via an Epson colour LCD projection monitor. The ACC interface comprised a Liquid Crystal Display (known as the message centre) in the instrument cluster and a set of buttons inset into the steering wheel. A separate PC was used to drive this interface. Transducers connected to the steering, brake and accelerator send signals to an Acorn Archimedes RISC PC. Bespoke software inside the Acorn interprets the signals to position the driver's trajectory along 
the motorway. Data were recorded every half a second by the simulator software, including: speed, position on the road, and distance from other vehicles. The simulator was equipped with a two-way intercom that allowed communication between the drivers and the experimenter.
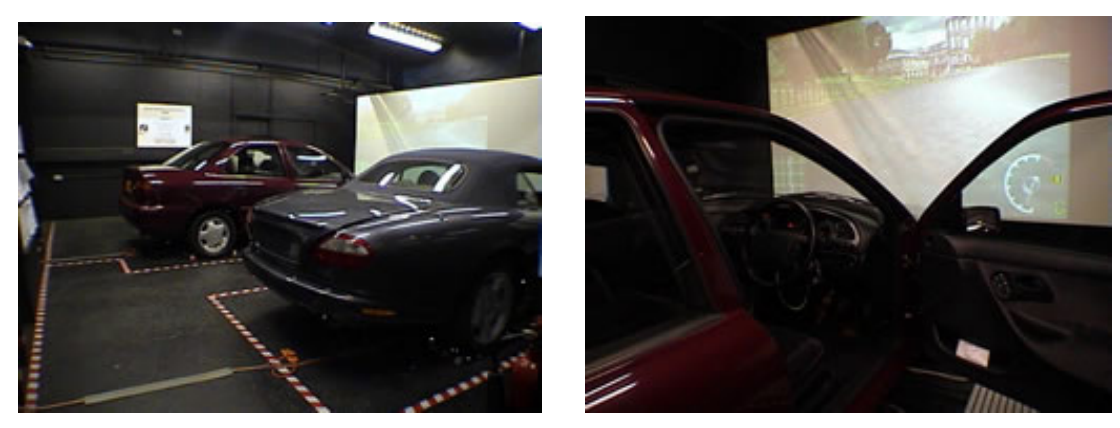

Figure 1 - Brunel Driving Simulators: Ford Mondeo and Jaguar X type

\subsubsection{Materials}

\subsubsection{Conceptual Model Building Exercise}

The participants were given instructions on the procedure of the experiment and the manual for the ACC system. Drivers' pictorial representations of ACC would be a window into their CMs and should indicate whether they match the system image as well as the experimenter's and designers' CM. Drivers completing a drawing task of their conceptual models of ACC over time may therefore lend insight into their overall CM of the system.

To aid in the completion of the conceptual model drawing task, they were given instructions for their task and fourteen features and functions of the system on post-it notes which were:

- ACC on, ACC off

- Driver

- Following

- Cruising

- Resume

- Set speed

- Set gap

- Stand-by

- $\quad$ LCD feedback: Speed =

- LCD feedback: Gap = 
- Driver override accelerate (cancel)

- Driver override: Brake (cancel)

- (distraction features Anti-Crash Control System and GPS Navigation)

Of these features, there were two were distraction features (Anti-Crash Control System and GPS (Global Positioning Satellite) Navigation) in the sense they did not belong in the functioning model of the ACC.

\subsubsection{Trust Scale}

The trust scale originally designed by Muir and Moray (1996) and also employed by Stanton and Young (2005) was selected for the present study. The trust questionnaire rated ten different sub-components of trust (competence, predictability, dependability, responsibility, responsibility over time, faith, ACC responds accurately, trust in display, overall degree of trust, and confidence in own rating). In addition they were given a diary response form where they were asked to describe their driving experience with the ACC. In regards to the diary responses, the categories that defined the search and analysis of the diaries were: driving experience with the ACC; how much trust was placed in the system in for each day and drive; what the participants' feelings were in regards to the ACC system, what the participants' feelings were towards the gap and the set speed function; did their level of trust in the set speed differ from their level of trust in the gap function.

\subsection{Procedure}

The participants were given the ACC manual to read which was also explained verbally by the experimenter. On day 1 , the participants were given a chance for a five minute practice drive with the ACC engaged so that they could familiarise themselves with the ACC. The participants were then to drive manually for five minutes, which was their control task. After the control task the participant was to complete the experimental trial where they were to drive with the ACC engaged for five minutes. In the unreliable conditions, the ACC would malfunction as soon as the car exceeded $50 \mathrm{Mph}$, leading the car to suddenly accelerate.

Group 1 was subjected to reliable automation during the ten days. Group 2 was subjected to five days of unreliable automation (sudden acceleration) and five days of reliable automation at a random pattern. This translated to a $50 \%$ level of reliability over the ten days. Group 3 was exposed to unreliable automation during the ten days $(100 \%$ unreliable). At the end of all the driving trials on each day, the participants completed a drawing task. They were given the instructions on how to draw a conceptual model of the system with an example from the experimenter. After they completed the drawing task they were asked to write down their thoughts and explain their model. The participants were then to complete the trust scale and a diary of their experience of the drive in regards to the ACC and their level of trust in the system. This procedure was completed everyday for ten days, except the practice task. Finally, at the end of the ten days, an interview was conducted to gauge more of an in depth insight into the participants' feelings and behaviour during the experiment which was recorded and transcribed. A debriefing statement was also constructed to inform the participants at the end of the 
experiment the aims of the study and that the malfunctions were induced by the experimenter.

\section{$3 \quad$ RESULTS and DISCUSSION}

\subsection{Trust Scale}

\subsubsection{The reliability of ACC and Drivers' self-reported trust scores}

The scale is comprised of 10 individual sub-scales (see Materials section) thus multidimensional composite score was derived by taking the mean of 10 sub-scales.

Figure 2 presents the overall median ratings of trust for each of the three groups over the ten days. Group 2 and 3 (semi-reliable and unreliable automation) display an inappropriate level of trust by reporting a moderate rating of trust overall even though the system was unreliable. The reliable group (group 1) however had a quite high rating of trust, which is arguably appropriate since the group was not exposed to any unreliable automation. To highlight the inappropriateness of the trust ratings of group 2 and 3 , Figure 2 also indicates a hypothesised appropriate level of self-reported trust scores between the three groups. Regarding the level of reliability that drivers were exposed to, then, one may have expected that trust was rated high as found in the reliable group but then trust should have decreased below a moderate level, such as 'quite low' and 'not at all' level of trust for group 2 and 3. 


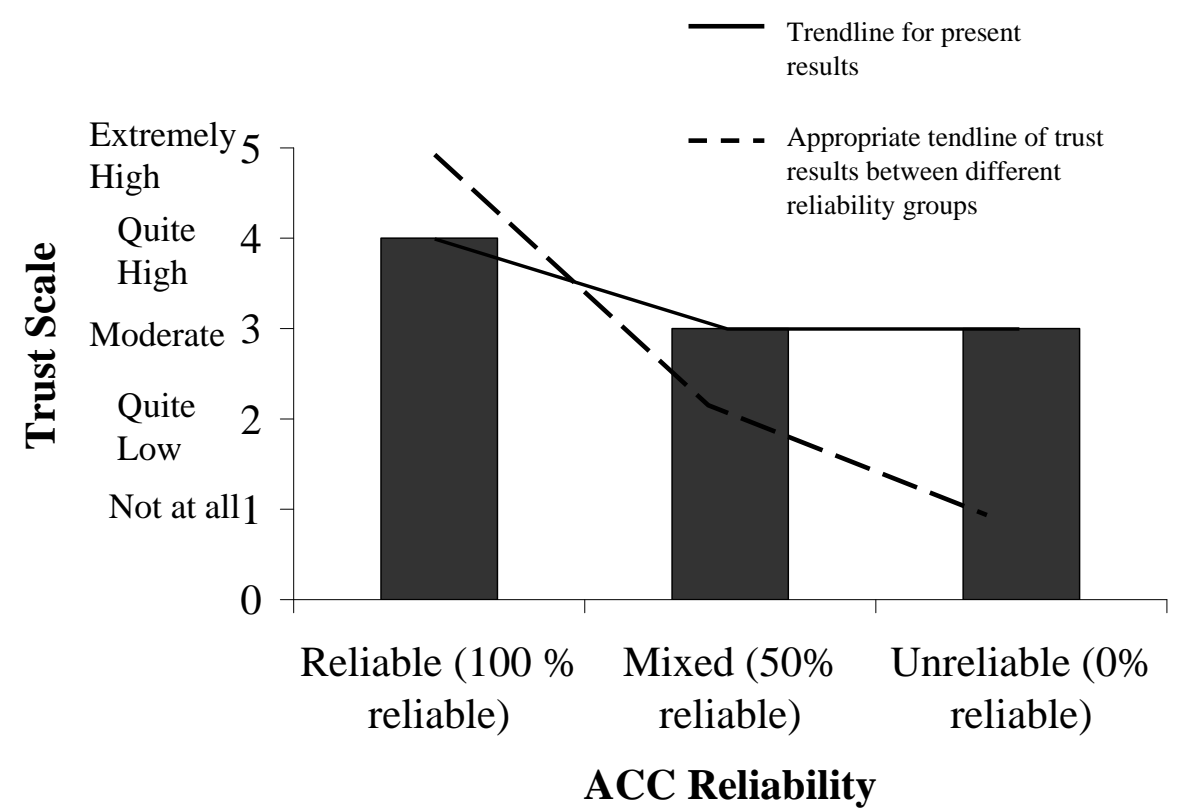

Figure 2 A histogram of the median composite trust score over ten days for the three groups of reliable automation; reliable, semi-reliable, unreliable

A Kruskal-Wallis test was performed on the data for each of the three groups for every one of the 10 days to determine whether there was a difference between the trust scores between the three groups (reliable, semi-reliable, and unreliable) over the ten days. There was a significant difference of trust between the three groups on day seven $(H=7.0, d f=2$; $\mathrm{p}<0.05$ ). This was further tested by a post hoc test, where for the present study the absolute value of the planned comparisons have to exceed the critical value of 6.56 . The only pair wise comparison that achieved this was between the reliable group and the unreliable group on day 7.

Group 2 and 3 still rated trust as moderate despite the high degree of unreliability (50 $\%$ and $0 \%$ ). Thus group 2 and 3 adopted an inappropriate level of trust. It is possible, particularly in group 2, that participants felt there were not enough failures to qualify the system to be of low reliability but there were enough failures to qualify as not entirely reliable. Therefore the drivers could not conclude that the system is not entirely trustworthy. This uncertainty, or 'dissonance,' a theory forwarded by Festinger, 1957(cited in Harmon-Jones and Mills 1999) may have produced such ratings.

Group 3 illustrated an automation bias since they still rated trust as moderate, thus over time drivers are still recalibrating their trust to inappropriate levels. Rudin-Brown and Parker's (2004) on-road study demonstrated that trust increased (pre-test trust scores versus post-test trust scores) in the ACC despite unreliable automation in their last 
driving condition, which is arguably also inappropriate. Hence it could be suggested that drivers recalibrating their trust to inappropriate levels in regards to automation reliability is a similar finding by both studies.

\subsubsection{Level of drivers' self-reported trust scores over time}

Below, figure 3 demonstrates the composite median scores taken from the trust scales for each day. Figure 3 illustrates that over the ten days, trust rises significantly (as demonstrated by the Friedman test below) for the reliable automation group from day 4 onwards. The drivers' self -reported trust scores for the reliable group hover around a moderate level, then there is a slight transition phase (and some fluctuation) around days 4,5,6. The drivers' self-reported trust scores are consolidated from day 7 onwards however. Figure 3 (page 15) hypothesised trust to be a discrete variable. Arguably, the trust ratings for the reliable group (figure 34) demonstrates tentative evidence for a 'stepup' function from day 3-5 and day 7, especially in light of the significant Friedman test below. However trust ratings for the semi-reliable and unreliable automation group stays much the same. From day 1-4 all trust results for both unreliable automation groups are the same at a moderate level. The semi-reliable automation group trust results creep slightly on day 5 to a score of 3.5 trust rating (moderate-quite high). However this then drops to a moderate rating from day 6,7,9\&10, falling to a quite low rating on day 8 . Interestingly group 3 , the unreliable group rate a 'moderate' level of trust throughout the ten days.

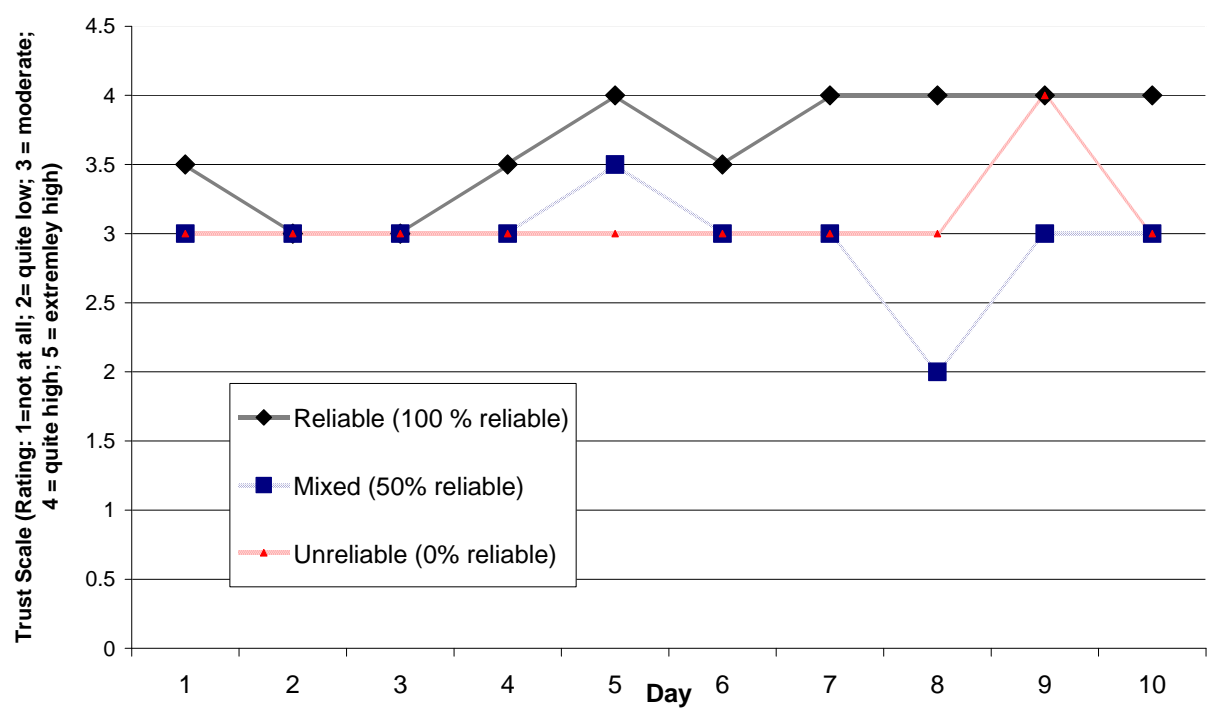

Figure 3 A Line graph of the median composite trust scores over ten days for all three groups 
A Friedman test was performed assess whether there was a difference of trust over time, which revealed that there was a significant difference of trust within the group 1 (reliable) condition (Chi-square $=26.2$; $\mathrm{df}=9$; $\mathrm{P}<0.05$ ). This was further tested by a post hoc test which revealed differences in driver's subjective ratings of trust over time. For instance, days 1, 2, 3, and 4 drivers' self-reported levels of trust significantly differ with those for days 5-10. Hence the hypothesis is supported as there were differences between driver's subjective ratings of trust over the ten days. Therefore it seems that in this instance there is evidence that drivers' level of self-reported trust to differ over time in a reliable condition.

\subsubsection{Trust Calibration}

The trust results in the present study differed from Rudin-Brown and Parker's (2004) finding. Their study exhibited an increase in trust ratings, despite the malfunction in their final driving condition, which arguably made the ACC system unreliable in the final condition. The present study only demonstrates the rise of trust ratings in the reliable automation condition, group 1.

The results of this investigation have implications for models that involve trust, especially if these models have been forged after one-off short-term experimentation. Vehicle automation and driver behaviour models that incorporate the trust variable as an influencing factor on performance should take into account that with time and experience, people's models of the system are made more realistic, which in turn recalibrates their trust. So trust may be 'moderate' seeing as that's no more than what these drivers normally expect of ACC within the ten days of driving with a malfunctioning ACC system, but with time this level of trust may increase or decrease appropriately to match the corresponding reliability of the system since future studies may illustrate that drivers' conceptual models will become clearer with experience.

\section{Drivers' conceptual models (DCM’s)}

\subsection{AGNA results}

The drivers' pictorial representations functioned as their conceptual model of the ACC system. They were surrogate models (see Young 1983) of the ACC system rather than mapping models, since they offered basic representations of the system function rather than a deep mapping explanation of the core technology. In order to calculate the day of consolidation of drivers' conceptual models (DCM) and trust, it was thought that a DCM and trust became consolidated once the model and level of trust started to be replicated after a certain day. For instance if a DCM was different from day one till day five but then after day six the model was portrayed to be exactly the same from day six onwards then day six was considered to be the day of consolidation. Accordingly, Group1's conceptual models tended to be consolidated by day 4 . To relate these results back to the trust results; the reliable group consolidated their models of the system on day 4 and drivers' subjective ratings of trust rose between day 3-5, (fluctuating on day 5-7) but stayed the same between day 7 and day 10. Hence drivers conceptual models fluctuate at first, becoming consolidated and not subject to change (regardless of being correct or not). Similarly drivers' subjective ratings of trust are not static on the first few days either 
and are subject to calibration, thus it increases. After day 7, however, drivers' selfreported trust arguably becomes consolidated also. This could be because once the drivers consolidate mentally their conceptual model of the ACC system this allows them to address their emotions in regards to their subjective ratings of trust in the system. It can be argued that perhaps in order for trust to be consolidated the drivers must consolidate their conceptual models of the system first rather than vice versa.

A novel analysis approach was taken based on graph theory using a software tool known as AGNA (Applied graph \& Network analysis) which is designed for social network analysis. Network analysis is a set of mathematical methods that assumes that features of a network model (nodes) that communicate the importance of the property of that feature (i.e. how central it is to the network, the higher the level of centrality the more it is considered to be a core feature of the entire network).

The idea was that each feature in a conceptual model would function as a node and an AGNA analysis would reveal the links between each feature within the conceptual model (i.e. whether the set-speed feature was linked, via arrows drawn, to the set-gap feature). Furthermore AGNA could denote the level of 'centrality' (referred to as the sociometric status) of each feature which illustrated how important each feature was in the sense of how many times it was linked to the other nodes. The more links between one node (ACC feature and others) then the higher level of centrality it would have. For example if the set-speed feature was linked to not only the set-gap feature but to two other features such as the following and cruising feature, while the set-gap feature was linked to one other feature, say LCD feedback of gap, then AGNA would calculate the level of links, producing the sociometric status between each feature/node which would reflect the degree of centrality of that node. Thus since set-speed (hypothetically) had more links to other features then this would make the set-speed feature more important in the drivers' conceptual model.

Figures 4-7 illustrate how the AGNA produces visual network construction from a sample conceptual model of one participant (that is a typical construction of the other models and AGNA outputs). Figure 4 and 6 are samples of one of the participant's conceptual model for day 1 and 10. Figures 5 and 7 are the corresponding visual representations of the network for the participant's conceptual models created by AGNA after measuring the sociometric status for each node. The figures illustrate how the links between the features change from day 1 through to day 10. There is a rich and complicated network construction on day 1 but a more simplified network by day 10 due to a reduction in links between features. 


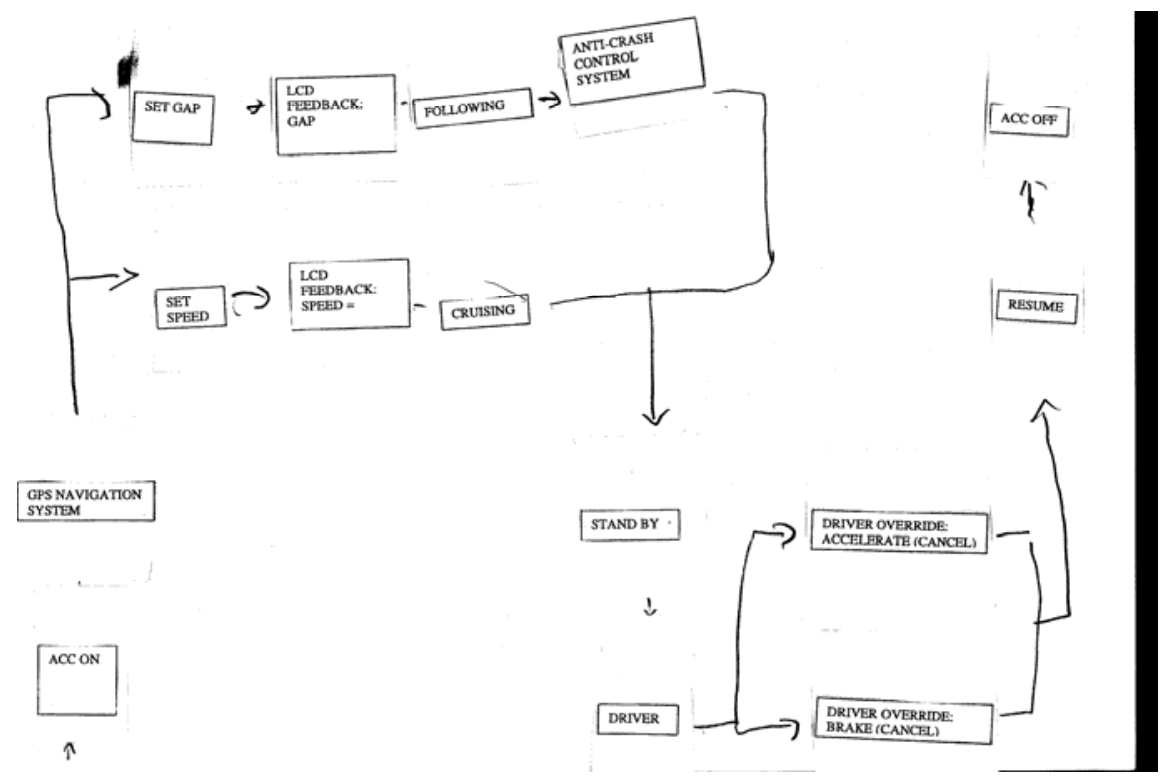

Figure 4 Participant 2 Conceptual model for day 1 


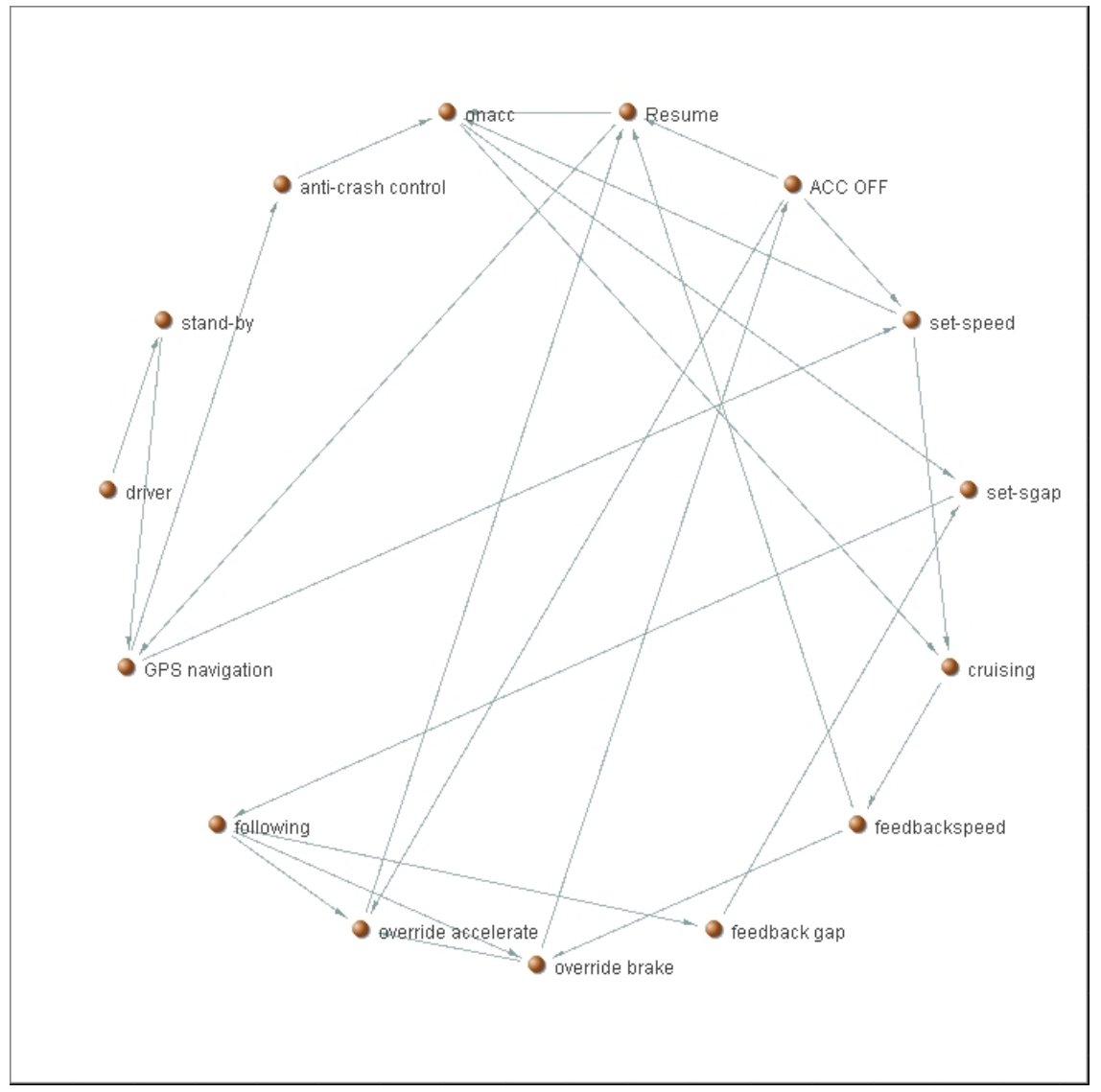

Figure 5 Participant 2 visual network for group 1(reliable) day 1

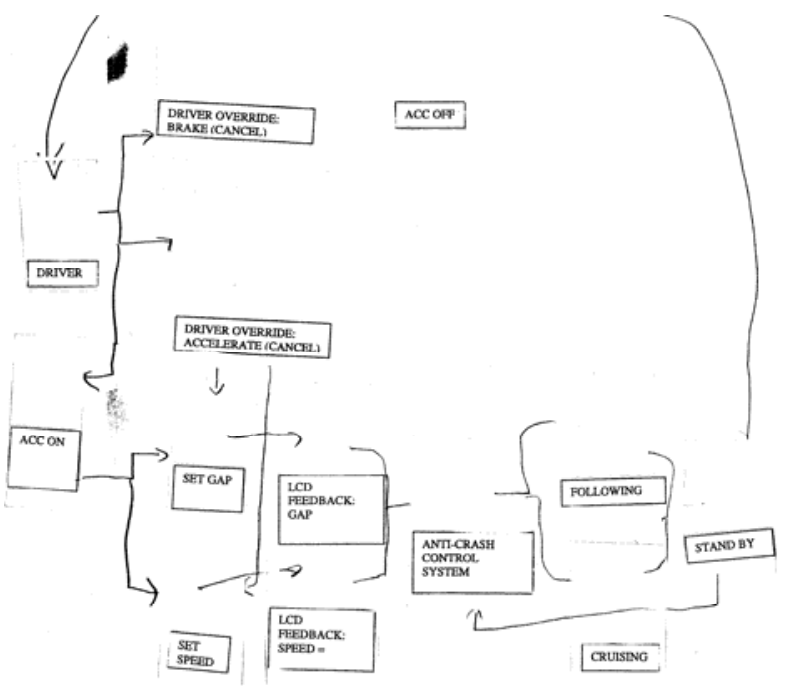


Figure 6 Participant 2 Conceptual model for day 10

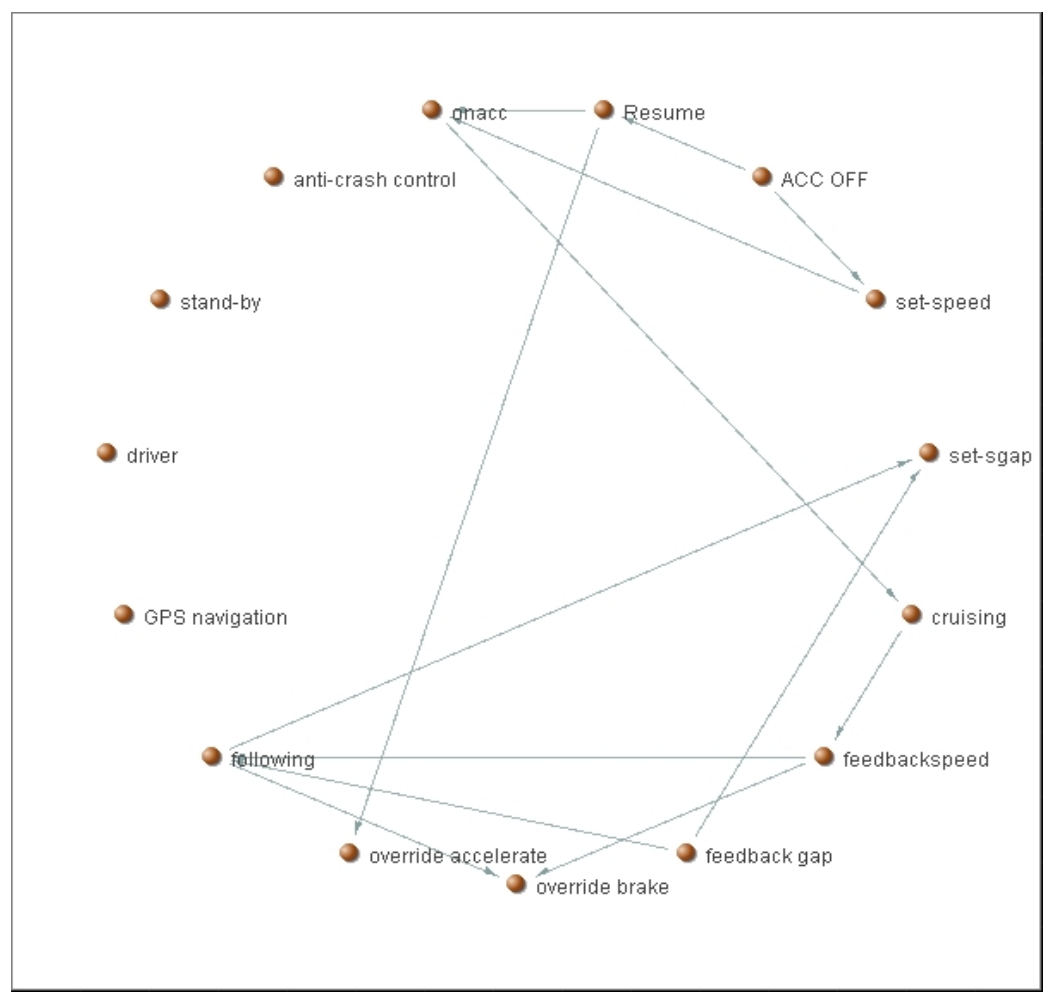

Figure 7 Participant 2's conceptual model network for day 10 (reliable automation, group 1)

The first stage of the analysis was to produce a 'baseline' model (which was the experimenter's model of the system based on the designers' model) and it functioned as a guideline model (see Figure 8 below). The guideline model was analysed using the AGNA software in order to assess the level of sociometric status to denote the level of centrality for each feature. The results for the level of centrality of the guideline model are presented below in Table 1 


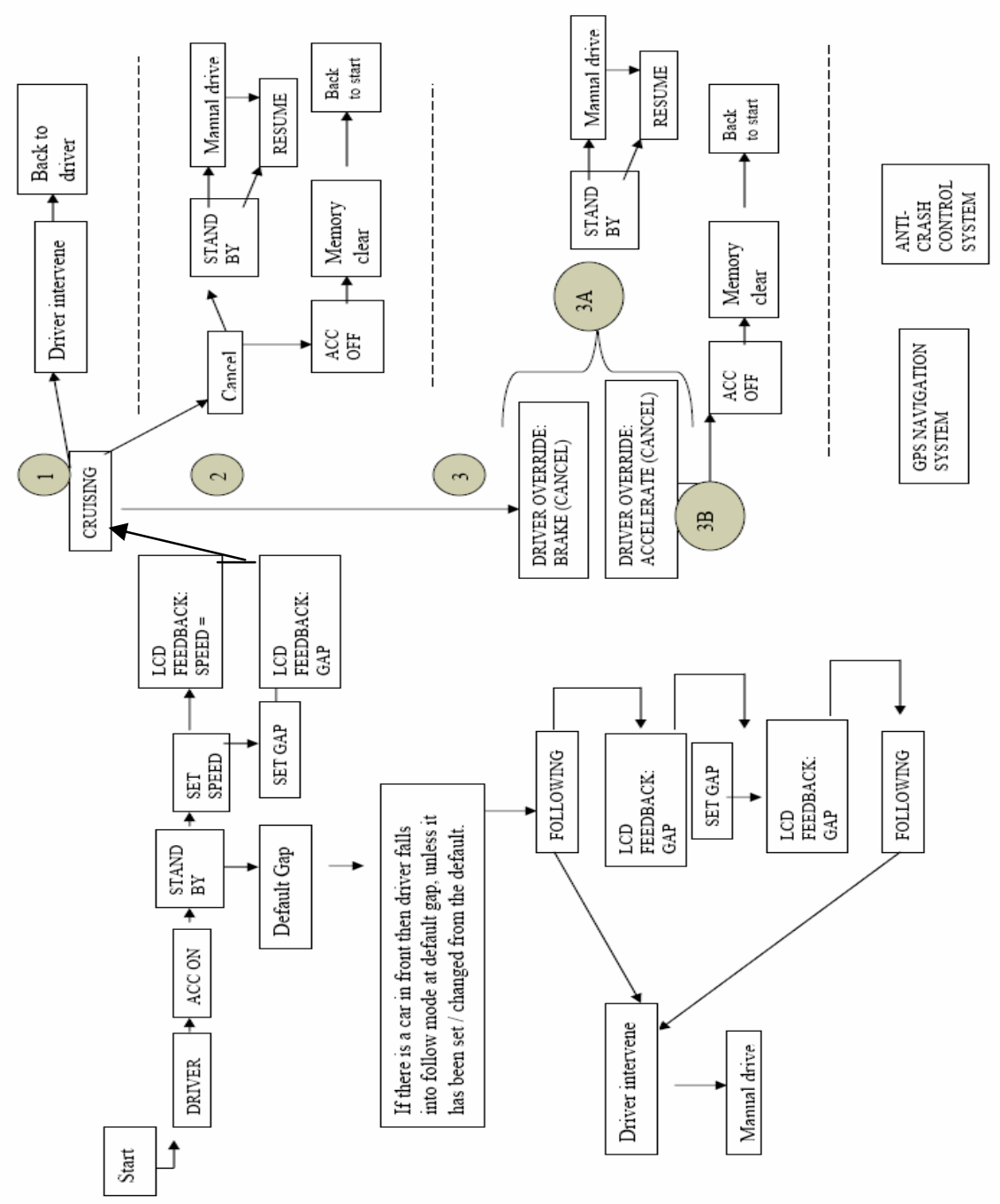

Figure 8 Guideline conceptual model of the system

Table 1 Distribution of sociometric status of the guideline model

\begin{tabular}{|l|l|}
\hline ACC Feature & Sociometric Status \\
\hline Stand-By & 0.33333334 \\
\hline Driver Intervene Warning & 0.1904762 \\
\hline Following & 0.1904762 \\
\hline Feedback Gap & 0.1904762 \\
\hline
\end{tabular}




\begin{tabular}{|l|l|}
\hline Manual Drive & 0.14285715 \\
\hline Memory Clear & 0.14285715 \\
\hline Acc Off & 0.14285715 \\
\hline Set-Speed & 0.14285715 \\
\hline Set-Gap & 0.14285715 \\
\hline Cruising & 0.14285715 \\
\hline Override Brake & 0.14285715 \\
\hline Override Accelerator & 0.14285715 \\
\hline Cancel & 0.14285715 \\
\hline Back To Start & 0.0952381 \\
\hline Resume & 0.0952381 \\
\hline Default Gap & 0.0952381 \\
\hline Driver & 0.052380953 \\
\hline Start & 0.052380953 \\
\hline Acc On & 0.04761905 \\
\hline Feedback Speed & 0.04761905 \\
\hline Anti-Crash Control & 0.0 \\
\hline GPS Navigation & 0.0 \\
\hline
\end{tabular}

The results show that for the guideline model the highest mean level of centrality $(0.33)$ assessed via the sociometric status was the stand-by feature. This is also reflected by figure 8, where the guideline model represents how the stand-by function is quite central to the system since this mode function allows the driver to resume or set-speed and even cancels the cruise mode and return to 'stand-by'.

The second stage of the analysis was to compare the drivers' conceptual models with the baseline model. The conceptual models were analysed via AGNA software to assess the sociometric status for each of the fifteen features for day 1 conceptual models and day 10 models in order to view whether ten days made a difference to the drivers conceptual models for each group. The mean sociometric status for each feature of the conceptual models for each group is presented in table 2 (day 1) and table 3 (day 10).

Table 2 The overall mean centrality for each of the fifteen features of the conceptual models for each group for day 1

\begin{tabular}{|l|l|l|l|}
\hline $\begin{array}{l}\text { Day 1 Feature of } \\
\text { ACC (node in the } \\
\text { AGNA network) }\end{array}$ & $\begin{array}{l}\text { Group 1 } \\
\text { sociometric status }\end{array}$ & $\begin{array}{l}\text { Group 2 } \\
\text { sociometric status }\end{array}$ & $\begin{array}{l}\text { Group 3 } \\
\text { sociometric status }\end{array}$ \\
\hline Resume & 0.2 & 0.1 & 0.2 \\
\hline
\end{tabular}




\begin{tabular}{|l|l|l|l|}
\hline ACC OFF & 0.3 & 0.2 & 0.1 \\
\hline set-speed & 0.2 & 0.2 & 0.2 \\
\hline set-gap & 0.2 & 0.2 & 0.1 \\
\hline cruising & 0.2 & 0.2 & 0.1 \\
\hline Feedback speed & 0.2 & 0.2 & 0.2 \\
\hline Feedback gap & 0.2 & 0.1 & 0.1 \\
\hline override brake & 0.2 & 0.2 & 0.1 \\
\hline override accelerate & 0.2 & 0.1 & 0.1 \\
\hline following & 0.2 & 0.2 & 0.2 \\
\hline GPS navigation & 0.1 & 0.1 & 0 \\
\hline driver & 0.1 & 0 & 0 \\
\hline stand-by & 0.1 & 0 & 0 \\
\hline anti-crash control & 0.1 & 0 & 0.1 \\
\hline ACC on & 0.2 & 0.1 & 0.2 \\
\hline & & &
\end{tabular}

Table 3 The overall mean centrality for each of the fifteen features of the conceptual models for each group for day 10

\begin{tabular}{|l|l|l|l|}
\hline $\begin{array}{l}\text { Day 10 Feature of } \\
\text { ACC (node in the } \\
\text { AGNA network) }\end{array}$ & $\begin{array}{l}\text { Group 1 } \\
\text { Level of centrality } \\
\text { (sociometric status) }\end{array}$ & $\begin{array}{l}\text { Group 2 } \\
\text { Level of centrality } \\
\text { (sociometric status) }\end{array}$ & $\begin{array}{l}\text { Group 3 } \\
\text { Level of centrality } \\
\text { (sociometric status) }\end{array}$ \\
\hline Resume & 0.1 & 0.2 & 0.1 \\
\hline ACC OFF & 0.2 & 0.1 & 0.2 \\
\hline set-speed & 0.2 & 0.1 & 0.2 \\
\hline set-gap & 0.1 & 0.1 & 0.2 \\
\hline cruising & 0.4 & 0.2 & 0.2 \\
\hline Feed back speed & 0.2 & 0.2 & 0.2 \\
\hline feedback gap & 0.2 & 0.1 & 0.1 \\
\hline override brake & 0.2 & 0.1 & 0.2 \\
\hline override accelerate & 0.2 & 0.1 & 0.1 \\
\hline following & 0.3 & 0.2 & 0.3 \\
\hline GPS navigation & 0 & 0 & 0 \\
\hline driver & 0.2 & 0.1 & 0.2 \\
\hline stand-by & 0.1 & 0.1 & 0.2 \\
\hline Anti-crash control & 0.1 & 0 & 0.1 \\
\hline ACC on & 0.2 & 0.1 & 0.1 \\
\hline
\end{tabular}

The results highlight three key points. Firstly, the guideline model did not match that of day 1 and day 10 between the three groups. Secondly, the results show that all three groups differed in sociometric status for the key feature of ACC (Stand-by) as ascertained by the guideline model. Finally, it can be argued that the drivers in all three groups were demonstrating an incorrect conceptual model of the system. Inspection of the sociometric status reflects that the drivers' conceptual models for each group differed over time and were influenced by reliability of the ACC, which supports the third hypothesis.

Initially, the present study was exploring the hypothesis that there would be a difference between the conceptual models for each group which was confirmed. Since the drivers conceptual models did not match that the guideline model, it then became of interest to examine this further and the 'distraction' functions which were the Anti-Crash- 
Control (ACCS) or General Positioning Satellite features (GPS navigation) were assessed to see whether they were included in any of the conceptual models.

\subsection{Erroneous Inclusion of Distraction Items (ACCS and GPS navigation)}

In order to assess whether the participants were constructing the correct conceptual models of the ACC system it was deemed appropriate to include two 'distraction' features within the list of ACC features for the conceptual model drawing task. GPS navigation and ACCS were deemed suitable as 'distraction' functions. GPS is a route navigation system and had no link to the ACC system at all while the ACC manual warns the drivers that the ACC system is not an Anti-crash control system. For this reason, if participants included either of these 'distraction' features in the conceptual models then this was considered incorrect.

The findings suggest that no matter what level of automation reliability, the ACCS feature was included in 33\% of the models, despite the ACC manual alerting the drivers that the system was not an anti-crash control system. More drivers included the ACCS feature in their model than the GPS navigation feature which was only included in $11 \%$ of the conceptual models overall. It could be possible that the drivers forgot the manual's warning that the ACC was not ACCS feature, however drivers' diary reports and interview responses highlighted that the drivers in question were convinced that the ACC was also a collision avoidance device.

When drivers were questioned during the interviews as to why they included the ACCS system, many reported because the "car slowed me down when I came up behind another car". The drivers were confusing the gap control function with the notion of collision-avoidance systems. Besnard et al (2004) postulates that when two consecutive situations occur due to random co-occurrence sometimes they can reinforce incorrect conceptual models and increase confidence in executing inappropriate actions. In this case, an event and cognition was wrongly paired; the 'gap' function performing its role of reducing the speed once it was in following mode to match the gap settings was paired with the notion that the non-existent ACCS function would slow the drivers down. When the drivers experienced collisions, they reported in their diaries "the ACCS function was malfunctioning". This induced confirmation bias which influenced the drivers to construct a flawed conceptual model of the ACC system. Ultimately the knowledge of the system could have been reinterpreted by the drivers to fit the model that drivers had of the situation, which maintained the invalid representations of the system.

At present the drivers' conceptual models are not matching those of the designers or researchers. This highlights that better design solutions may be warranted and perhaps driver re-training and education may be needed in regards to the driver's building the correct conceptual model of the system. Part of the mismatch arises from the fact that the designers and researchers argue that the ACC system is not a collision avoidance system (e.g., Richardson et al., 1997). However, because the ACC system can arguably exhibit collision avoidance type behaviour at certain times but not consistently. It is debatable that the behaviour of the gap function can be confused with the idea of an ACCS system. Simulator training or on-road training with a co-driver may help drivers build appropriate trust and conceptual models of the system. 


\subsection{Diaries and Interviews}

A descriptive summary of the diaries and interviews for each participant is presented in table 4.

Table 4 Descriptive summary of the overall issues and themes that were raised through the Diary entries and Interviews by each group

\begin{tabular}{|l|l|l|l|}
\hline Overall issues and themes & G1 & G2 & G3 \\
\hline $\begin{array}{l}\text { The ACC is good for motorway } \\
\text { driving }\end{array}$ & $*$ & $*$ & \\
\hline Difficult to hand over 'control' & & $*$ & \\
to a 'computer' & & \\
\hline $\begin{array}{l}\text { Drivers exhibit a lack of } \\
\text { situational awareness when } \\
\text { driving with ACC }\end{array}$ & $*$ & $*$ & $*$ \\
\hline $\begin{array}{l}\text { Drivers reported that the ACC } \\
\text { was badly designed and had } \\
\text { very low usability and } \\
\text { suggested re-design issues for } \\
\text { the interface, especially the gap } \\
\text { function and its settings }\end{array}$ & $*$ & & \\
\hline $\begin{array}{l}\text { Initial difference between trust } \\
\text { levels within the set-speed and } \\
\text { set- gap function but trust } \\
\text { equalising after a few days of } \\
\text { driving }\end{array}$ & $*$ & $*$ & \\
\hline $\begin{array}{l}\text { Drivers were very disappointed } \\
\text { with the ACC system }\end{array}$ & & & \\
\hline $\begin{array}{l}\text { The ACC system was useful } \\
\text { and easy to use }\end{array}$ & $*$ & & \\
\hline
\end{tabular}

There are two themes raised by most of the drivers through the diaries and interviews that are worth discussing, which are how the drivers felt that the ACC system is only suitable for motorways and how the gap settings were not suitable. This finding has also been reported through a longitudinal on-road study by Francher, Ervin, and Bogard (1998 cited in Van Der Heijdden and Wiethoff 1999). They concluded that the driver's age and his/ her driving style related to the choice of one of the three gap settings (1.0 seconds, 1.4 seconds and 2.0 seconds). In addition they recommended that and in order to cater for different driver preferences, a range of gap time settings was needed.

The idea that the set-gap settings were too restrictive was also echoed by participants from the present study, mainly from group 1 (reliable), as they suggested that the gap settings were not "big enough" and gap increments should be proportional to the speed. Some drivers even suggested that the ACC should have ultimate control over the gap function so that drivers cannot adjust the gap settings.

\section{Conclusion}

A ten day study was carried out to examine drivers' level of trust and conceptual models of the ACC system. The present study has illustrated that not only do the subjective 
ratings of trust rise over time, but that drivers have a propensity to trust the system inappropriately as well as consolidating an incorrect conceptual model of ACC in a very short time.

This may have implications for models that involve drivers' trust and that have been forged after one-off short-term experimentation. Such models should factor in the idea, that with time and experience, trust will slightly fluctuate and will be re-calibrated, thus the level of trust within the system will change over time. In addition the drivers' conceptual models do not match that of designers or the experimenters' model, especially since ACC is confused with ACCS, thus better design solutions may be warranted.

A tentative suggestion for a possible design solution to reduce the risk of the ACC being employed as a ACCS device would be re-design the ACC system so that the adaptive headway feature (gap control) becomes a 'level of automation' (LOA) through 'Adaptive Automation' (AA) approaches (see Sheridan and Verplank, 1978; Parasuraman, Sheridan and Wickens, 2000; Kaber and Endsley, 2004 and Endsley and Kaber, 1999). AA is often referred to allocation of control between a human operator and computer over time. The difference between the AA and LOA approaches is that LOA involves fixed function assignments defining the degree to which a task is automated and AA involves dynamic control allocations which can be automated or manual, and vary over time (Kaber and Endsley, 2004). The overall aim is to improve human operator performance of complex systems by maintaining operator involvement in the control of the system (Kaber and Endsley, 2004).

Such an approach would mean that the driver is presented in essence with conventional cruise control (CC) with the option of engaging the gap headway setting (rather then the present automatic engagement of gap headway settings as soon as the driver sets the speed). If the system experiences too many 'driver intervention' warnings during the drive (normally evoked if a vehicle jumps into the lane ahead and the ACC cannot cope with the close proximity of the sudden presence of the lead vehicle), the system then would warn the driver that the ACC is reverting to CC mode and gap headway is not available until the driver chooses to engage the gap feature again. Such a design solution is offered with caution however since switching between modes of automation levels could prove to be more harmful than helpful to drivers. Especially since there would be a need to provide feedback on what mode the ACC is in (ACC or CC) and the results of Stanton and Young's (2005) study lead them to discourage the use of a HUD (higher-up-display) to relay information in a textual format to the driver.

It is possible that future smart technologies, such as Advanced Driver Assisted Systems (ADAS) prototypes may already address the shortcomings of present day marketed ACC systems. Carsten (2005 cited in Underwood 2005) maps the next developmental phases of ACC systems, soon to be ADAS, which are;

- Initiating a STOP\&GO feature allowing the ACC to drive down to $0 \mathrm{~km} / \mathrm{h}$

- Addition of forward collision avoidance

- Enabling the ACC negotiate all car following situations and allowing automated longitudinal control

- Adding assistance systems for lateral control such as lane changes,

- Finally full autonomous driving will be achieved with the addition of a "crossing Assistant" to assist drivers at intersections 
The advent of such fully autonomous systems such as ADAS into consumer vehicles may not be without its drawbacks. It is possible such a system will evoke a high trust level from the drivers as well as an incorrect model of the system since drivers will be out of the loop and could potentially hamper development of a rich situational awareness and understanding of the system. For instance Harris and Harris (2004) suggests that very high levels of automation may increase driver underload, which from a safety perspective, is potentially as unfavourable as high levels of workload (Young and Stanton 1997; Stanton and Young, 2001). These studies suggest that there is performance degradation when drivers are mentally underloaded. For instance, simulated studies of unreliable ACC systems show the negative impact on drivers' response time to reclaim control (Stanton, et al, 1997). A possible explanation lies in the theory of malleable attentional resources (Young and Stanton, 2005), where mental resources 'shrink' to accommodate demand reduction. In light of this, it can be suggested that such highly automotive systems would further displace the driver from the driving control loop and reduces drivers' response times in the event of ADAS malfunctions.

Furthermore, it can be argued that combining comfort and assistive system such as Adaptive-Cruise-Control, Anti-Crash-Control System and GPS, for example, as part of one overall vehicle assistant system (as designers plan to do so through ADAS) may actually induce further driver over reliance and enhance inappropriate conceptual models. Norman (1990) suggests that the effectiveness of conceptual models is influenced primarily by the system interface. One option to discourage potential inappropriate driver reliance and conceptual models then would be to design separate interfaces for each device (GPS, ACC and ACCS) rather than combine all these intelligent automation devices together through one interface. It should be noted that separate interfaces may not be enough to discourage driver over reliance on such systems or offer clarity of conceptual models of such devices. Since, arguably, it was the adaptive behaviour of the gap function slowing the vehicle to match headway settings that re-enforced the notion that the ACC was an ACCS device. As a final concluding remark however, just because eventually such systems can be functionally combined, does not mean that designers should do so. Not least before, such systems or proto-types are evaluated to determine the potential impact on comfort, usability and road safety (Stewart and Harris 2003).

\section{Acknowledgements}

The author would like to express her thanks to the Brunel University. The present research was conducted for Brunel University, using the Brunel University Simulator and Brunel University resources and has no affilation to TRL (Transport Research Laboratory) other than corresponding address for the author.

\section{REFERENCES}

Bainbridge, L. 1982 Ironies of automation. In J. Rasmussen, K. Duncan, \& J. Neplat (Eds.), New technology and human error (pp 151-157). New York: Wiley

Besnard, D. Greathead, D \& Baxter, G. 2004. When mental models go wrong: co-occurrences in dynamic, critical systems. International Journal Of Human -Computer studies 60, 117-128 
Carsten, O. 2005. Mind over matter: Who's controlling the vehicle and how do we know In Traffic and Transport Psychology. Theory and Application. Pages 231-242. Edited by Underwood, G. Published by Elsevier Ltd. The Boulevard, Langford Lane. Oxford 0X5 1GB UK ISBN 0080443796

Endsley, M. R. and Kaber, D. B. (1999) Level of automation effects on performance, situation awareness and workload in a dynamic control task Ergonomics, 42, 462-492.

Fancher, P. Ervin, R. and Bogard, S. 1999. Behavioural reactions to advanced cruise control: results of a driving simulator experiment. In R.E.C.M. Van der Heijden \& M. Wiethoff Automation of car driving. Exploring societal impacts and conditions (pp 103-104). TRAIL research school Delft.

Harris, D. \& Harris, F. (2004)Evaluating the transfer of technology between application domains: a critical evaluation ofthe human component in the systemTechnology in Society 26 PP 551565

Harmon-Jones, E and Mills, J. 1999 Cognitive Dissonance: Progress on a Pivotal Theory in Social Psychology (pp 11). Washington, DC : American Psychological Association

Hoedemaeker, M. \& Brookhuis, K.A. (1998). Behavioural adaptation to driving with adaptive cruise control (ACC). Transportation Research Record Part F, 1, 95-106.

Hoedemaeker, M \& Kopf, M. (2001). Visual Sampling Behaviour when driving with adaptive cruise control. In proceedings of the ninth international conference on Vision in Vehicles. Australia, August 19-22.

Kaber D. B and. Endsley, M.R. 2004. The effects of level of automation and adaptive automation on human performance, situation awareness and workload in a dynamic control task Theoretical Issues in Ergonomics Science. March-April 2004, vol. 5, no. 2, 113-153

Kantowitz, B.H. Hanowski, R.J. \& Kantowitz, S.C. 1997. Driver acceptance of unreliable traffic information in familiar and unfamiliar settings. Human Factors, 39(2), 164-176.

Lee, J.D. \& See, K.A. 2004. Trust in automation: Designing for Appropriate Reliance. Human factors, Vol. 46, No. 1, Spring. pp 50-80.

Lee, J. And Moray, N. 1992 Trust, control strategies and allocation of function in human -machine systems. Ergonomics 35(10), 1243-1270

Lewandowsky, S. Mundy, M. Tan, G, P.A. 2000. The dynamics of trust: comparing Humans to automation. Journal of Experimental Psychology: Applied. 6(2), 104-123

Moray, N. Inagaki, T \& Itoh, M. 2000. Adaptive automation, trust, and self-confidence in fault management of time-critical tasks. Journal of Applied Experimental Psychology, 6(1) pp4458

Muir, B.M. 1994. Trust in automation: part 1. Theoretical issues in the study of trust and human intervention in automated systems. Ergonomics 37(11), 1905-1922 
Muir B.M. and Moray, N. 1996. Trust in automation. Part II. Experimental studies on trust and human intervention in a process control simulation. Ergonomics 39, 429-461.

Norman, D.A. 1990. The 'problem' with automation: Inappropriate feedback and interaction, not 'over-automation'. Philosophical Transactions Of The Royal Society Of London, B327, 585593.

Parasuraman, R., Sheridan, T. B. and Wickens, C. D. 2000, A model of types and levels of human interaction with automation, IEEE Transactions on Systems, Man and

Cybernetics, 30, 286-297.

Parasuraman, R. and Riley, J. 1997. Humans And Automation: Use, Misuse, Disuse, Abuse. Human factors, 39 (2), 230-253

Preece, J Rogers, Y and Sharp, H. 2002. Interaction design: beyond human-computer interaction (pp 39-55) John Wiley \& Sons, Inc.

Rasmussen, J., Petjerson, A. M. \& Goodstein, L. P. (1994). Cognitive Systems Engineering. New York: Wiley.

Richardson, M., Barber, P., King, P., Hoare, E., \& Cooper, D. (1997). Longitudinal driver support systems. Proceedings of Autotech '97 (pp. 87-97). London: IMechE.

Rudin-Brown, C.M. Parker, H.A. 2004. Behavioural adaptation to adaptive cruise control (ACC): implications for preventive strategies. Transportation research Part 2(7), 59-76

Sayer, J.R, Francher, P.S., Bareket, Z, \& Johnson, G.E. (1995). Automatic target acquisition autonomous intelligent cruise control (AICC): Driver comfort, acceptance, and performance in highway traffic, SP-1088, Human Factors in Vehicle Design: Lighting, seating and Advanced Electronics.

Sheridan, T. B. and Verplank, W. L. 1978, Human and computer control of undersea teleoperators (Cambridge, MA: MIT Man-Machine Laboratory).

Stanton, NA. Young M. \& McCaulder, B. 1997. Drive -by-wire: the case of driver workload and reclaiming control with adaptive cruise control. Safety Science Vol 27, NO. 2 / 3, pp.149159.

Stanton, N.A. and Young, M.S. (2005). Driver Behaviour With Adaptive Cruise Control. Ergonomics. Vol. 48, No.10, PP.1294-1313

Wilde, G.J.S. 1982. The theory of risk homeostasis: implications for safety and health. Risk Analy., Volume 2, 209-225.

Young, R. M (1983) Surrogates and Mappings: Two Kinds Of Conceptual Models For Interactive Devices. In Gentner, D and Stevens, A.L. Mental Models (pp 35-52) Lawrence Erlbaum Associates, Cambridge, England

Young \& Stanton . 2001. Out of control. New Scientist 172 (2315) pp 44-47 